\title{
Market of stocks during crisis looks like a flock of birds
}

\author{
Bahar Afsharizand ${ }^{1,2}$, Pooya, H. Chaghoei ${ }^{2}$, Amirhossein A. Kordbacheh ${ }^{1}$, Andrey Trufanov ${ }^{3}$ \\ and Golamreza Jafari $4,5 *$ \\ 1 Department of Physics, Iran University of Science and Technology, Tehran, Tehran,Iran; \\ afsharizand@gmail.com, akordbacheh@iust.ac.ir \\ 2 Center for Complex Networks \& Social DataScience, Shahid Beheshti University, Tehran, Tehran, Iran; \\ pooya1991@gmail.com \\ 3 Irkutsk National Research Technical University, Irkutsk, Russia ; troufan@gmail.com \\ 4 Department of Physics, Shahid Beheshti University, Tehran, Tehran,Iran ; gjafari@gmail.com \\ 5 Department of Network and Data Science, Central European University, Budapest, Hungary \\ * Correspondence: gjafari@gmail.com
}

\begin{abstract}
According to its inner property, a crisis in the financial market can be considered as a collective behavior phenomenon. Through the prism of collective behavior, the crisis does not happen if the companies are independent of each other. In this work, cooperative movement processes in a stock market are investigated in a manner similar to that Vicsek first described collective behavior for self-propelled entities. To this end, a phase space is defined as the one in which the return of volume of transactions versus return of price is represented with each share in each day corresponding to a unique point in the space. The findings of the observation show that during times of crisis, the phase space is limited with the vector velocity of shares in the same direction. In contrast, on a regular day, the phase space is entirely accessible, with vector velocity aligned randomly. Moreover, in line with the Vicsek model, an order parameter is introduced, which evaluates the cooperative effects for the shares so that the higher the value of this parameter, the stronger the collective behavior of the shares.
\end{abstract}

Keywords: Collective behavior, Vicsek model, Financial crisis

\section{Introduction}

The emergence of the collective behavior observed in many systems like schools of fish or flocks of birds actually guarantees the chances of survival for the whole system [1,3? ]. In financial stock markets, staying in the community of similar entities makes any company exposed to lesser risks and thus boosts the chances of survival in financial crises rather than being non-cooperative. The question arises as to what causes the collective behavior of the stocks of the companies to be? One might assert with a different notion that if there were no financial markets and the stocks of the companies were not in the structure, there would be no financial crisis. Similarly, the collective movement in schools of fish causes accumulates of predators around the group. In fact, in spite of getting stronger to be in a group for each member, it would itself be a major factor to spread a problem of each member within all the groups. One of the most important issues in financial markets $[33,34]$ is the identification and forecasting of crises with intense market downturns [4-6]. So far, this has been considered in previous studies with a variety of approaches [7,8]. Cooperative movement of complex systems such as schools of fish, flocks of birds, or insects with myriad actors that move in the same direction without any leadership, can be considered as a brilliant example of collective behavior in nature [9]. Prominently illustrated the complexity of a flock of migrating geese and its principal distinction from Boeing 747-400 with its millions of components. Also, the authors discussed how this complexity might be 
studied quantitatively and understood entirely by applying network theory. From the physics point of view, many efforts have been made to identify and understand the general principles governing cooperative processes [10-12]. While studying Collective order in these systems, in which interaction between actors is of short-range type for over the latest 25, various models have been suggested to understand and formulate large-scale features of group movements [13-15]. Nevertheless, in spite of many cases, the general principles have not been entirely clarified so far: perhaps the most important reason for the observed ambiguity is that systems are not in equilibrium. Hence, standard theorems and the statistical mechanics techniques being accurate for description long-range order in equilibrium systems, bring no use to understand the onset of seemingly similar phenomena in large groups of conscious actors, self-propelled organisms, or systems of particles. The virtue of privacy makes it difficult or even impossible to analyze the collective behavior of investors. Thus it seems of value to examine the movement of stocks which manifest on investment processes in economics. So, a framework has to be adopted to examine whether or not collective behavior occurs during critical market days. In order to study the cooperative phenomena in non-equilibrium systems, numerous models have been suggested. Among them, the prominent one developed by Vicsek et al in 1995 [16] with its rule that prescribes to align directions that particles are traveling in their neighborhood has been considered as the most basic simulation platform for further advances in the domain. Later this platform has been extended in different aspects, e.g. in Czirok model Vicsek, Barabasi and Czirok,1999 observed symmetry breakdown and ordering of self-propelled particles in one-dimension [17] that contradict previous analytical conclusions severely. In 2000 Levin $t$ al proposed a model [18] in which he enhanced the scope of Vicsek by taking into account both attractive and repulsive forces. Buhl model [19], 2006 which implies a modification of velocity weighting of the particles approves rapid transition from the disordered movement of individual actors to highly aligned cooperative motion. In contrast to the formless and point-like particles described within the Vicsek model, Peruani attributed forms and packing coefficients to the actors [20].There are much more models based upon Vicsek platform such those of Chate [21], Cocker-Smiley [22], Galam [23], and [24]. Here, we analyze the observations Vicsek framework proposes which is justified because of the basic feature of this model.

\section{Data Description}

We used Standard\&Poor's500(S\&P500) stock market data, which presents activities of 500 large companies listed on stock exchanges in the United States. The analyzed data consist of the 465 stocks computed from 2007 to 2019 mid-year and comprise opening price information, closing price, high price, low price, and adjusted on a daily basis.

\section{Vicsek Model}

The model deal with a system of self-propelled particles. The basic idea in the Vicsek model $(\mathrm{VM})$ is that at each time step, each actor prefers to move In the average direction its neighbors align but distorted with some noise due to imposed disorder [25]. In other words, all the agents of the system strive to follow their neighbors. However, the particles might make mistakes while evaluating their neighbors' trajectory, which is considered as "noise". Within the general introduction of the two-dimensional Vicsek scope, one assumes that VM considers particles on a two-dimensional plane having periodic boundary conditions. In this model, each particle is characterized by two coordinate of position and velocity in space. The model implies that the particles move with equal speed and simultaneously the direction of the velocity vectors of particles changes as a function of time. Thorough mathematical description of the model defines a circular neighborhood area $U_{n}\left(r_{0}, t\right)$ with the radius $r_{0}$, centered at the $\mathrm{n}$-th particle position $X_{n}(t)$ and containing the number of close neighbors $K_{n}(t)$ so that outside the $U_{n}\left(r_{0}, t\right)$ no interactions occur for the $\mathrm{n}$-th particle. Then the average velocity of the neighbors of the particle $n$ is calculated by: 


$$
V_{n}(t)=\frac{1}{K_{n}(t)} \sum_{j: x_{j} \in U_{n}} v_{j}(t)
$$

where $v_{j}(t)$ denotes the velocity of $\mathrm{j}$-th particle inside $U_{n}\left(r_{0}, t\right)$.

Consequently, the time progress for the velocity $v_{n}(t)$ of n-th particle, pertinent angle $\theta_{n}(t)$, which defines the velocity direction, and the particle position $r_{n}(t)$, all being exposed to angle noise of amplitude $\eta$ can be written as:

$$
\begin{gathered}
\theta_{n}(t+\Delta t)=\text { Angle }\left[V_{n}(t)\right]+\eta \xi_{n}(t), \\
v_{n}(t+\Delta t)=v_{0} e^{i \theta_{n}(t+\Delta t)}, \\
x_{n}(t+\Delta t)=x_{n}(t)+v_{n}(t+\Delta t) \Delta t .
\end{gathered}
$$

At any time $t$, the degree of the order in a system might be assessed through the order parameter $\Phi_{\eta}(t)[26,27]$, which is expressed by:

$$
\Phi_{\eta}(t)=\frac{1}{N v_{0}}\left|\sum_{n=1}^{N} v_{n}(t)\right| .
$$

This parameter ranges within $[0,1]$ so that random distribution of the velocity vectors in isotropic case gives zero, while 1 corresponds to entire alignment of the vectors. It is of value that transition from a disordered state to large-scale ordered dynamic mode of the agents depends on the density of those and noise amplitude in the system.

\section{Results and discussion}

Prior to simulate the collective behavior of agents in the stock market on the Vicsek model platform, it is necessary to introduce a coordinate framework to identify and clarify the cooperative environment. Regarding this closing prices price and trading volumes from daily stock market data of S\&P500 [28] were quantified which actually led to a two-dimensional problem Within this X-Y coordinate system, each market instrument might be characterized by two parameters: closing price $C P_{t}$ and trading volume $V_{t}$ at time interval between $t$ and $t-1$, as Eq. 6 portrays:

$$
X_{t}=C P_{t}-C P_{t-1} \quad Y_{t}=V_{t}-V_{t-1}
$$

where $t$ takes just discrete values $0,1, \ldots, t-1, t, t+1, \ldots$.

If follow Eq. 6, the X-axis and the Y-axis of the coordinate system show the daily final price difference and the difference of trading volumes respectively for two successive days both. As mentioned above density of particles is one of the fundamental factors in collective behavior within VM.

It seems of value to transform the stocks so to set them on a similar scale and thus to reduce influence of daily market trends in the model. That might be done through normalization of $X_{t}$ and $Y_{t}$ to the mean value in some time period, e.g. previous 20 days.

$$
X_{t}=\left(X_{t}-\mu_{x}\right) / \sigma_{x} \quad Y_{t}=\left(Y_{t}-\mu_{y}\right) / \sigma_{y}
$$

Using the stock coordinate in the new phase space, one can attribute a "speed" parameter for a daily stock as follows:

$$
V_{t}=\left(X_{t+1}-X_{t}\right) \mathbf{s}_{x}+\left(Y_{t+1}-Y_{t}\right) \mathbf{s}_{y}
$$


where $\mathbf{s}_{x}$ and $\mathbf{s}_{y}$ are unit vectors along $X$-axis and $Y$-axis respectively. Also in the above definition the variations of the quantities $X_{t}$ and $Y_{t}$ in time unit are calculated.

Fig. 1 exhibits the velocity vectors of all one-day stocks for three critical market days in comparison with those of normal functioning of the market. It is seen that during non-crisis period the stocks tend to move randomly whereas their more coordinated movement across the market indicates collective behavior on the days of crash. These six graphs show the velocity vector of 465 stocks, which according to the history of the market, have been referred to three ordinary days and three those of crisis. We may add that if model the process for other days of the crisis, collective behavior of stocks is clearly visible as well.

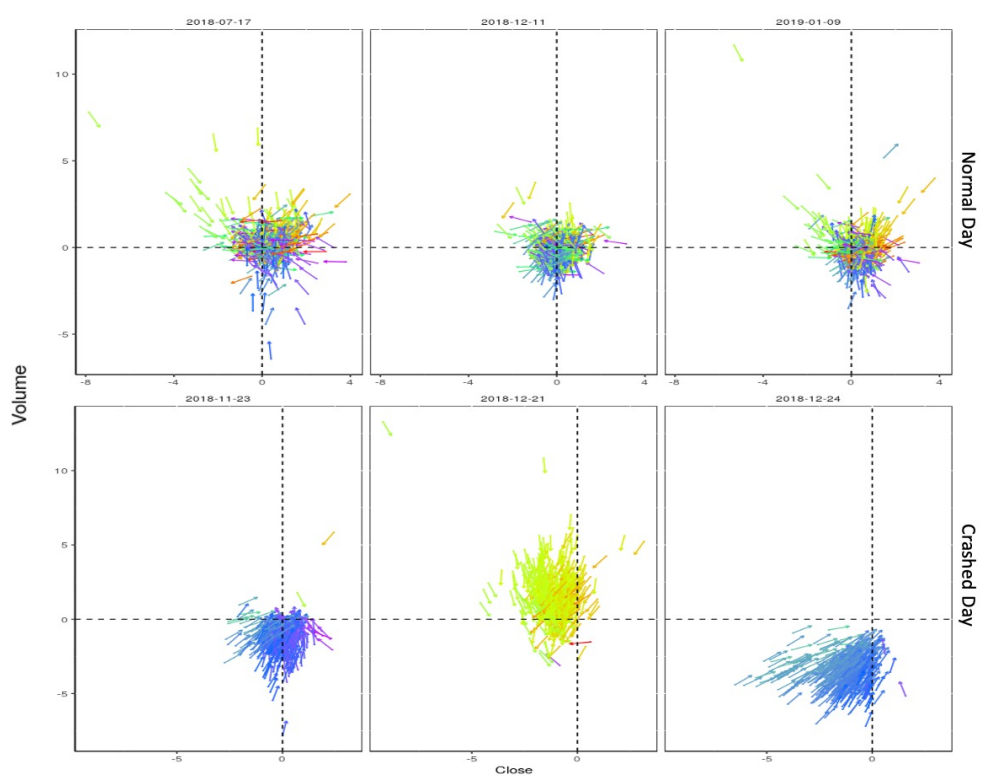

Figure 1. Collective Behavior of stocks in crisis. Comparison of stock movement for three critical days and for those away from crisis.

The results of our observations give evidence to conclude that there is collective behavior in the capital market.

Now, it deems significant to develop a daily metric which is related to the phenomenon in the market and then to compare (capture) their characteristics on different days.

Based on this new measurable value, and by monitoring its trend one can identify the days when cooperative processes occur and thus to predict the crisis. For convenience, we define local an $X-Y$ coordinate system for each company's stock and divide the $2 \mathrm{D}$ space into $\mathrm{m}$ parts. Therefore putting the "speed" vector into the center of the appropriate system will come to be in one of m sectors.

Further, to aggregate the case we examine all the pairs of shares together so that if $\mathrm{A}$ is in sector 1 and $B$ is in sector 2, the pairing brings stocks A and B into the sector (1-2).

performing the same procedure for all stocks and counting the number of different pair-stocks over $C_{m+k+1}^{k}$ combinations $(\mathrm{k}=2)$, we will find out how many shares have fallen in each coupled sector every day.

We consider sufficient to assign $\mathrm{m}=12$, and thus the number of coupled sectors is equal to 78 .

As shown in Fig 2 in most of the days, pair-stocks distribute in all possible pair-regions, and these are the days when stock space is severely restricted. The space in the above description actually refers to the 12 sectors where each component of the pair of shares comes into. On normal days, pair-shares can get correspond any of the 78 possible modes, but on the days of the crash, they get into a smaller 
number of sectors depending on the level of crisis.
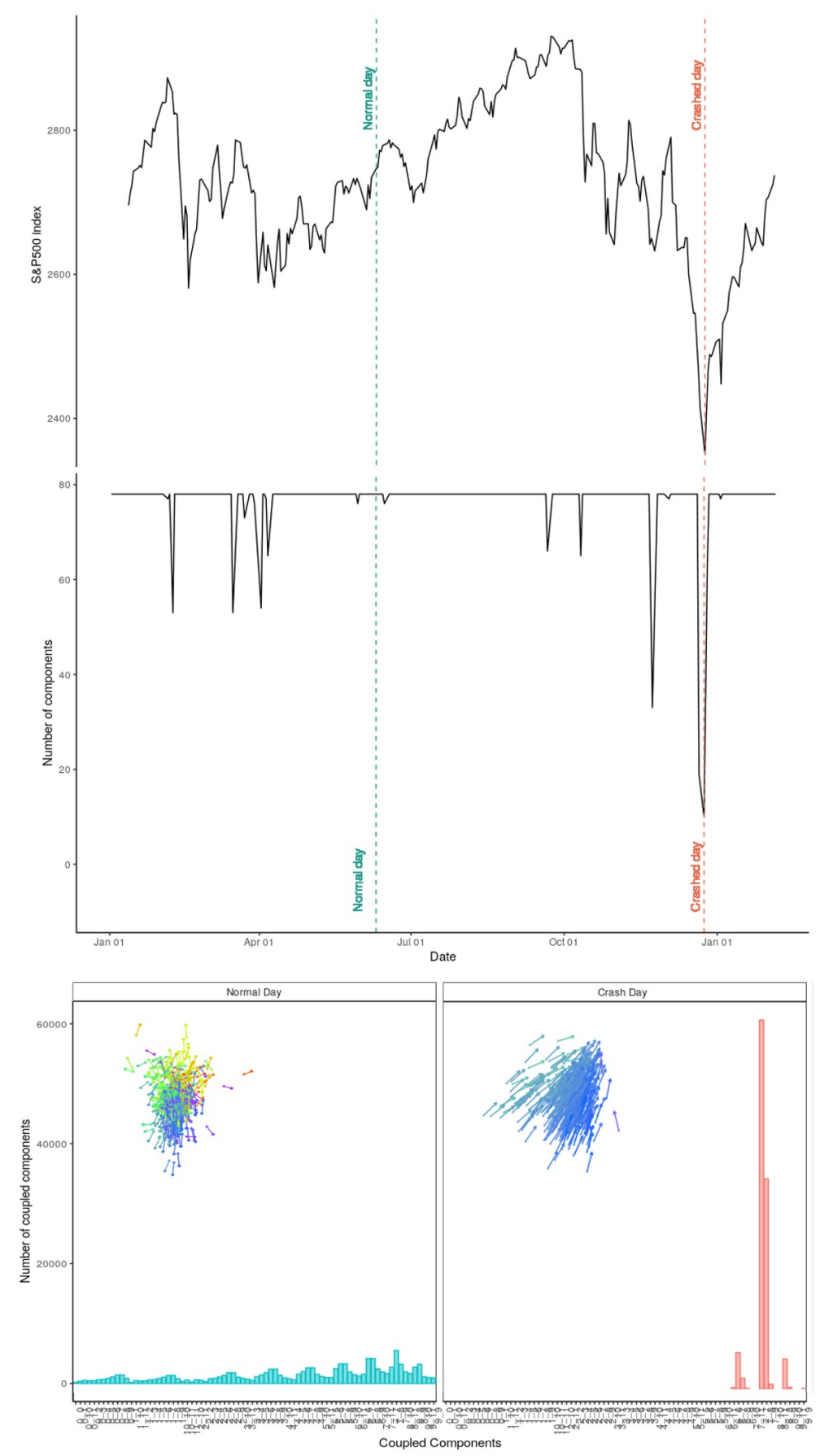

Figure 2. Bold the figure title. A: The historical trend of $S \& P 500$ index for 2018. B: The number of accessible pair component in each day of 2018 and compare them to the history of the index. C: Distributions of coupled components in normal day and on the day of crash.

Thus the pair-stock distribution over coupled sectors might be taken into account to indicate the coming crisis.

Preliminary investigations of the most recent emergence of collective behavior show that the market had been also experiencing severe fluctuations in the overall index those days.

Actually the observations justified our initial expectations of the collective phenomenon in the stock market during severe downturns. 
It is of sense to look for a metric in the new coordinate system similar to VM order parameter to indicate collective behavior that the more it declines, the more evidently cooperative effects manifest and so the more likely the crisis will happen.

With no doubt It is of sense to look for a metric in the new coordinate system similar to VM order parameter just to indicate collective behavior that the more it declines, the more evidently cooperative effects exude and so the more likely the crisis will happen.

In order to quantify the level of the order in collective motion, one might use a scalar parameter $\Phi_{\eta}(t)$ defined as:

$$
\Phi_{\eta}(t)=\left|\sum_{n=1}^{N} V_{n}(t)\right| .
$$

In any normal day when the directions of motions are completely random, $\Phi_{\eta}(t)$ is small enough whereas in a day of great crisis, $\Phi_{\eta}(t)$ reaches large value because many velocity vectors are aligned.
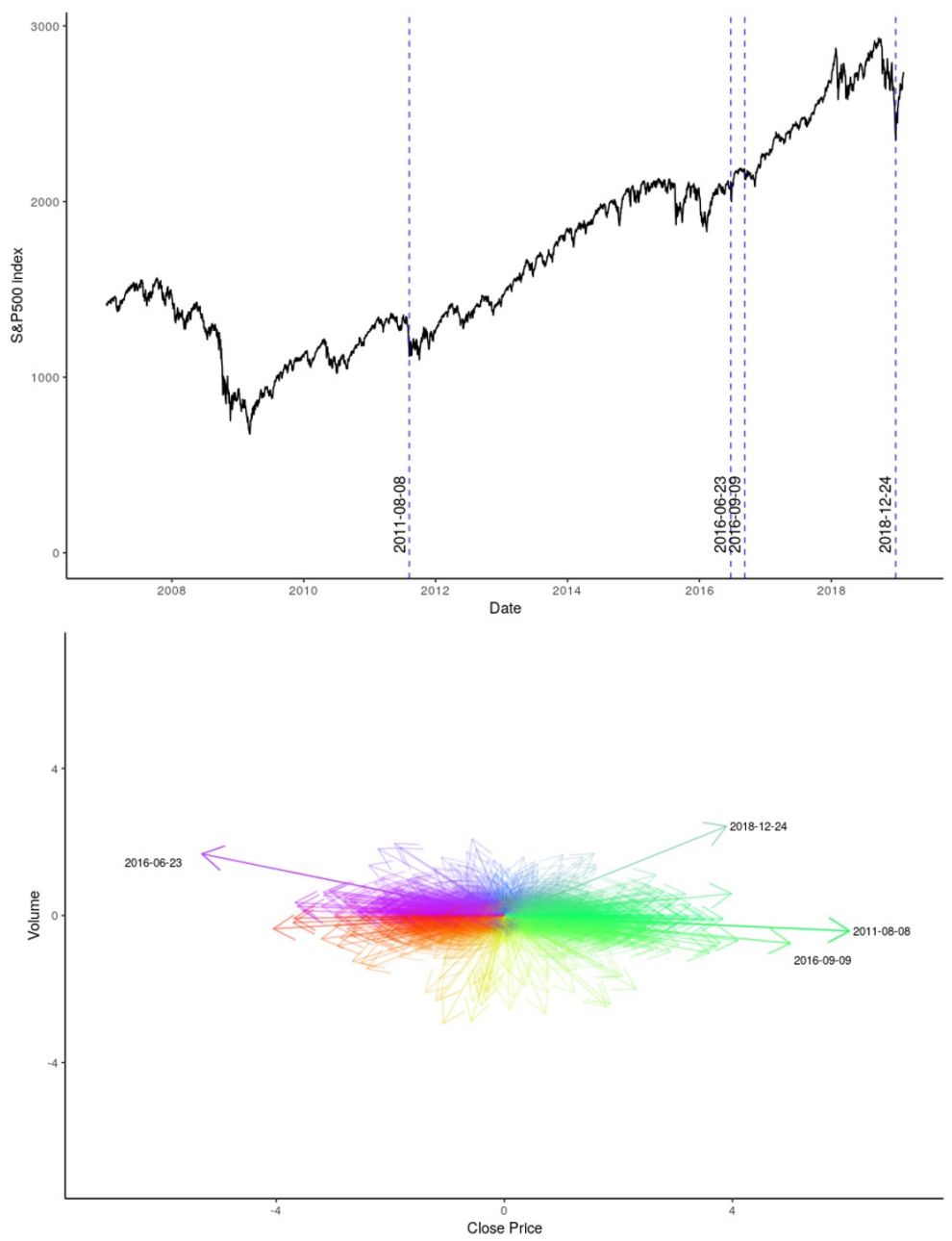

Figure 3. Bold the figure title. A: historical data of S \& P500 index trend from 2010 to 2019. B: The vectors of order parameter for all the days of 2010 to 2019 , and label some crash days.

\section{Conclusion}

According to the results, we can claim that in critical times of the market, all stocks have skin in the game.

The reason for this is that the occurrence of the stock crisis and the emergence of the collective behavior are strongly interconnected and interdependent We have been able to provide the 
implementation of the Vicsek approach for matching crisis and cooperative phenomenon in a stock market.

We maintained the framework of VM by defining a specific metric - order parameter based on summarizing the velocity vectors over all stocks per day and calculating the norm of the sum. Further, it was shown that this metric reaches its most enormous value on the days of the stock market crash. It is evident one can also use this order parameter to classify crises.

It is of value to underline that collective motion, such as considered within the Vicsek model, is not necessarily leading to stock alignment [29], and there might be patterns that imply collective behavior [30,31] or have a lag due to timing [32]. So it makes reason to modify VM for better identification, classification, and even prediction of the stock market crash.

An additional exciting result of this review is that, even though on December 24, 2018, as we pursued the largest merger in the stock market of $S \& P 500$, no subsequent crisis was noticed.

Surely, from one hand if decrypt stock market motion properly it might give some evidence of crisis in advance, on another hand this behavior is merely a reaction to the survival of the entire system in times of crisis.

Further experts need to discuss how to indicate the structure in which the crisis is occurring and how to define an order parameter to examine the structure near and beyond the crash.

Author Contributions: Conceptualization, GR. Jafari; methodology, Bahar Afsharizand and Pooya H. Chaghoei; software, Bahar Afsharizand and Pooya H. Chaghoei; validation, GR. Jafari and A. Trufanov; formal analysis, AA. Kordbacheh; investigation, Bahar Afsharizand and Pooya H. Chaghoei; resources, Bahar Afsharizand and Pooya H. Chaghoei; data curation, Bahar Afsharizand and Pooya H. Chaghoei; writing-original draft preparation, Bahar Afsharizand and AA. Kordbacheh; writing-review and editing, A. Trufanov; visualization, Bahar Afsharizand; supervision, GR. Jafari; project administration, GR. Jafari.

Funding: This research received no external funding.

Acknowledgments: We thank professor Tamas Vicsek for reading and useful discussion.

Conflicts of Interest: The authors declare no conflict of interest.

\section{References}

1. Lao P, Singh H. Herding behaviour in the Chinese and Indian stock markets. Journal of Asian Economics. 2011;22(6):495 - 506. doi:https:/ / doi.org/10.1016/j.asieco.2011.08.001.

2. Giardina I. Collective behavior in animal groups: Theoretical models and empirical studies. HFSP Journal. 2008;2(4):205-219. doi:10.2976/1.2961038.

3. Lukeman R, Li YX, Edelstein-Keshet L. Inferring individual rules from collective behavior. Proceedings of the National Academy of Sciences. 2010;107(28):12576-12580. doi:10.1073/pnas.1001763107.

4. Yang J, Hsiao C, Li Q, Wang Z. The Emerging Market Crisis and Stock Market Linkages: Further Evidence; 2006.

5. Wang Z, Yang J, Bessler DA. Financial crisis and African stock market integration. Applied Economics Letters. 2003;10(9):527-533. doi:10.1080/1350485032000100198.

6. Xu R, Chang J, Li C, Wang W. Research on the influence of equity pledge on stock price crash risk: based on financial shock of 2015 stock market crisis. Economic and Political Studies. 2019;7(4):480-505. doi:10.1080/20954816.2019.1667599.

7. Iren P. Behavior of Foreign Investors in the Malaysian Stock Market in Times of Crisis: A Nonlinear Approach. Journal of Asian Economics. 2018;60. doi:10.1016/j.asieco.2018.11.002.

8. Namaki A, Shirazi AH, Raei R, Jafari GR. Network analysis of a financial market based on genuine correlation and threshold method. Physica A: Statistical Mechanics and its Applications. 2011;390(21):3835 - 3841. doi:https://doi.org/10.1016/j.physa.2011.06.033.

9. Amaral, L A N , Ottino, J M . Complex networks - Augmenting the framework for the study of complex systems. Eur Phys J B. 2004;38(2):147-162. doi:10.1140/epjb/e2004-00110-5.

10. Toner J, Tu Y. Flocks, herds, and schools: A quantitative theory of flocking. Phys Rev E. 1998;58:4828-4858. doi:10.1103/PhysRevE.58.4828. 
11. Jung N, Kim P. Marginalized ordering and adaptive reaction time in bird flocks; 2019.

12. Saeedian M, Jamali T, Kamali MZ, Bayani H, Yasseri T, Jafari GR. Emergence of world-stock-market network; 2017.

13. Mishra S, Tunstrøm K, Couzin ID, Huepe C. Collective dynamics of self-propelled particles with variable speed. Phys Rev E. 2012;86:011901. doi:10.1103/PhysRevE.86.011901.

14. Szabó P, Nagy M, Vicsek T. Transitions in a self-propelled-particles model with coupling of accelerations. Phys Rev E. 2009;79:021908. doi:10.1103/PhysRevE.79.021908.

15. Jafari G, Shirazi AH, Namaki A, Raei R. Coupled Time Series Analysis: Methods and Applications. Computing in Science Engineering. 2011;13(6):84-89. doi:10.1109/MCSE.2011.102.

16. Vicsek T, Czirók A, Ben-Jacob E, Cohen I, Shochet O. Novel Type of Phase Transition in a System of Self-Driven Particles. Phys Rev Lett. 1995;75:1226-1229. doi:10.1103/PhysRevLett.75.1226.

17. Czirók A, Barabási AL, Vicsek T. Collective Motion of Self-Propelled Particles: Kinetic Phase Transition in One Dimension. Phys Rev Lett. 1999;82:209-212. doi:10.1103/PhysRevLett.82.209.

18. Levine H, Rappel WJ, Cohen I. Self-organization in systems of self-propelled particles. Phys Rev E. 2000;63:017101. doi:10.1103/PhysRevE.63.017101.

19. Buhl J, Sumpter DJT, Couzin ID, Hale JJ, Despland E, Miller ER, et al. From Disorder to Order in Marching Locusts. Science. 2006;312(5778):1402-1406. doi:10.1126/science.1125142.

20. Peruani F, Deutsch A, Bär M. Nonequilibrium clustering of self-propelled rods. Phys Rev E. 2006;74:030904. doi:10.1103/PhysRevE.74.030904.

21. Chaté H, Ginelli F, Montagne R. Simple Model for Active Nematics: Quasi-Long-Range Order and Giant Fluctuations. Phys Rev Lett. 2006;96:180602. doi:10.1103/PhysRevLett.96.180602.

22. Cucker F, Smale S. Emergent Behavior in Flocks. IEEE Transactions on Automatic Control. 2007;52(5):852-862. doi:10.1109/TAC.2007.895842.

23. Galam S. Modelling rumors: the no plane Pentagon French hoax case. Physica A: Statistical Mechanics and its Applications. 2003;320:571 - 580. doi:https:/ /doi.org/10.1016/S0378-4371(02)01582-0.

24. Chepizhko AA, Kulinskii VL. On the relation between Vicsek and Kuramoto models of spontaneous synchronization. Physica A: Statistical Mechanics and its Applications. 2010;389(23):5347 - 5352. doi:https://doi.org/10.1016/j.physa.2010.08.016.

25. Ginelli F. The Physics of the Vicsek model. The European Physical Journal Special Topics. 2016;225(11-12):2099-2117. doi:10.1140/epjst/e2016-60066-8.

26. Aldana M, Dossetti V, Huepe C, Kenkre VM, Larralde H. Phase Transitions in Systems of Self-Propelled Agents and Related Network Models. Phys Rev Lett. 2007;98:095702. doi:10.1103/PhysRevLett.98.095702.

27. Baglietto G, Albano EV. Nature of the order-disorder transition in the Vicsek model for the collective motion of self-propelled particles. Phys Rev E. 2009;80:050103. doi:10.1103/PhysRevE.80.050103.

28. Nasiri S, Bektas E, Jafari GR. The impact of trading volume on the stock market credibility: Bohmian quantum potential approach. Physica A: Statistical Mechanics and its Applications. 2018;512:1104 - 1112. doi:https://doi.org/10.1016/j.physa.2018.08.026.

29. Ozogány K, Vicsek T. Modeling the Emergence of Modular Leadership Hierarchy During the Collective Motion of Herds Made of Harems. Journal of Statistical Physics. 2014;158(3):628-646. doi:10.1007/s10955-014-1131-7.

30. Arenas A, Fernández A, Gómez S. Analysis of the structure of complex networks at different resolution levels. New Journal of Physics. 2008;10(5):053039. doi:10.1088/1367-2630/10/5/053039.

31. Pinter-Wollman N, Fiore S, Theraulaz G. The impact of architecture on collective behavior. Nature Ecology and Evolution. 2017;1. doi:10.1038/s41559-017-0111.

32. Nagy M, Akos Z, Biro D, Vicsek T. Hierarchical group dynamics in pigeon flocks. Nature. 2010;464(7290):890-893.

33. Yang, Xin and Zhao, Xian and Gong, Xu and Yang, Xiaoguang and Huang, Chuangxia Systemic Importance of China's Financial Institutions: A Jump Volatility Spillover Network Review Entropy, 2020;22

34. Memon, Bilal and Yao, Hong Structural Change and Dynamics of Pakistan Stock Market During Crisis: A Complex Network Perspective Entropy, 2019;21 
(C) 2020 by the authors. Licensee MDPI, Basel, Switzerland. This article is an open access article distributed under the terms and conditions of the Creative Commons Attribution (CC BY) license (http:/ / creativecommons.org/licenses/by/4.0/). 